ITM Web of Conferences 5, 00015 (2015)

DOI: $10.1051 /$ itmconf/20150500015

(C) Owned by the authors, published by EDP Sciences, 2015

\title{
Cell-based modeling of cell-matrix interactions in angiogenesis
}

\author{
Roeland M.H. Merks ${ }^{1,2, a}$ \\ ${ }^{1}$ Centrum Wiskunde \& Informatica, Life Sciences, Science Park 123, 1098 XG Amsterdam, The Netherlands \\ ${ }^{2}$ Mathematical Institute, Leiden University, Niels Bohrweg 1, 2333 CA Leiden, The Netherlands
}

\begin{abstract}
The self-organization of endothelial cells into blood vessel networks and sprouts can be studied using computational, cell-based models. These take as input the behavior of individual, endothelial cells, as observed in experiments, and gives as output the resulting, collective behavior, i.e. the formation of shapes and tissue structures. Many cell-based models ignore the extracellular matrix, i.e., the fibrous or homogeneous materials that surround cells and gives tissue structural support. In this extended abstract, we highlight two approaches that we have taken to explore the role of the extracellular matrix in our cellular Potts models of blood vessel formation (angiogenesis): first we discuss a model considering chemical endothelial cell-matrix interactions, then we discuss a model that include mechanical cell-matrix interactions. We end by discussing some potential new directions.
\end{abstract}

\section{Introduction}

Cell-based, mathematical models of angiogenesis aim to explain the formation of blood vessel sprouts and networks, from the behavior of individual, endothelial cells and associated cells. The aim of these models is twofold: (a) to help experimental biologists get new insights into angiogenesis and develop new hypotheses; and from a mathematical point of view (b) as a model system for exploring how simple, cell behavioral rules can give rise to complex multicellular patterns. Our earlier models showed how endothelial cells can self-organize into networks or sprouts [1-4], but they ignored a key component of many developmental mechanisms: the extracellular matrix (ECM). The ECM is an umbrella term for the extracellular materials that provide cells and tissues with structural support, including many proteins, e.g., collagen, fibronectin, elastin and proteoglycans, e.g. hyaluronic acid.

Apart from providing structural support, the ECM acts as a medium to transduce or store messages between cells. The chemical composition of the ECM material can act as a molecular signal itself, or it can bind growth factors that cells secrete. In addition to such chemical signaling, the ECM also acts as a medium for mechanical signaling. Cells can exert forces on the matrix by exerting active forces or simply by exerting pressure on adjacent tissues. The resulting strains and stresses can elicit cellular responses and may thus coordinate the collective behavior of cells locally or over longer distances. Whereas direct cell-to-cell signaling acts over short times and distances, ECM-mediated signaling might act over much longer times, by depositing a chemical signal and moving away, and over longer distances, by generating strain fields. Thus, apart as a mechanical support, the ECM plays a key role

ae-mail: merks@cwi.nl

This is an Open Access article distributed under the terms of the Creative Commons Attribution License 4.0, which permits unrestricted use distribution, and reproduction in any medium, provided the original work is properly cited. 
as a mediator of cell-cell communication, and therefore, it cannot be ignored in cell-based models of angiogenesis.

In this short review, we discuss two of our previous, explorative cell-based modeling studies of ECM-mediated cell-cell communication during angiogenesis $[5,6]$. The first study shows how local, proteolytic modification of the ECM can coordinate the behavior of endothelial cells during sprouting angiogenesis [5]; the second study [6] shows how mechanical cell-cell communication via the ECM can coordinate the formation of vascular-like networks.

\section{Model and Results}

Cell-based models simulate the motility of individual, biological cells and their response to signals from adjacent cells. They take as input the observed or hypothetical rules that the individual cells follow, i.e., the behavior of endothelial cells, and predict the collective behavior emerging from these rules: i.e., the blood vessel sprouts or blood vessel networks. Among the large variety of cell-based modeling techniques that exist our method of choice is the cellular Potts model (CPM). In the CPM cells can assume a variety of shapes and respond differently on one end of the cell than at the other, a feature that many of the phenomena that we study depend on.

The CPM represents cells on a regular lattice $\Lambda \subseteq \mathbb{Z}^{d}$; in the models presented here, $d=2$ and $\Lambda$ is a regular square lattice. Biological cells are represented as (usually, but not necessarily, connected) patches of lattice sites $\vec{x} \in \Lambda$ that have an identical cell identifier, or spin, $\sigma(\vec{x}) \in\{1, \ldots, n\}$, with $n$ the number of biological cells in the simulation. Each spin, $\sigma$ is also associated with a set of cellular properties, including $\tau \in\{0, \ldots, m\}$, a cell type, and $A_{T}$, the cellular volume around which the cells fluctuate. $\sigma(\vec{x})=0$ represents the extracellular space in the CPM. In the hybrid models presented in this short article, each lattice site is additionally associated with the local value(s) of one or more fields; e.g., a chemical field $c$ in the next section, or a strain field $\epsilon$ in Section 2.2.

The CPM evolves by attempting to move the boundaries between cells. A Metropolis algorithm iteratively selects at random a pair of adjacent lattice sites $(\vec{x}, \vec{x} \prime)$ and attempts to copy the spin $\sigma(\vec{x} \prime)$ into the adjacent site $\vec{x}$, in order to minimize a Hamiltonian $H>0$,

$$
H=\sum_{\left(\vec{x}, \vec{x}^{\prime}\right)} J\left(\tau(\sigma(\vec{x})), \tau\left(\sigma\left(\vec{x}_{\prime}^{\prime}\right)\right)\right)\left(1-\delta\left(\sigma(\vec{x}), \sigma\left(\vec{x}_{\prime}^{\prime}\right)\right)\right)+\lambda_{A} \sum_{s \in\{1, \ldots, n\}}\left(a(s)-A_{T}(s)\right)^{2} .
$$

The first term in the Hamiltonion represent adhesion, where the parameters $J \geq 0$ are the adhesive energies and are defined for each pair of cell types, and the Kronecker delta term selects the cell-cell interfaces. The second term constrains the area (or volume for $d=3$ ) of the cells; $\lambda_{A}$ is a Lagrange multiplier, and $a(s)=|\{\vec{x} \in \Lambda: \sigma(\vec{x})=s\}|$ is the actual area of cell $s$, i.e., the number of lattice sites it occupies. A copy attempt is accepted if $\Delta H \leq 0$, or with a probability $P(\Delta H)=\exp (-\Delta H / T)$ if $\Delta H>0$ in order to mimic membrane fluctuations. The time unit of the CPM is the Monte Carlo Step (MCS), consisting of as many copy attempts as there are sites in the lattice. In the models presented here, interactions with the external fields are included by modifying $\Delta H$ at the time of copying based on the local value or gradient of the fields, as explained in more detail in Sections 2.1 and 2.2.

\subsection{Chemical cell-ECM interactions}

In a first study on cell-matrix interactions [5] we explored how the extracellular matrix can help coordinate cell behavior during angiogenesis by means of purely biochemical mechanisms. In this model, inspired by, e.g., the work of Anderson and Chaplain [7] and [8], endothelial cells migrate 
from a parent vessel and attempt to migrate towards a tumor, guided by a steady-state gradient of vascular endothelial growth factor,

$$
c_{V}\left(x_{2}\right)=c_{V}(0) e^{-x_{2} / \lambda} .
$$

Here $c_{V}\left(x_{2}\right)$ is the concentration of VEGF along a vertical coordinate $x_{2}, c_{V}(0)$ is the concentration of VEGF at the tumor boundary, and $\lambda$ is the diffusion length, the distance over which the concentration of VEGF has reduced to $1 / e$ of $c_{V}(0)$.

The endothelial cells migrate up the chemoattractant gradient by preferentially extending their boundaries towards higher concentrations of the VEGF gradient. In the cellular Potts model, chemotaxis is usually implemented by introducing an extra change, $\Delta H$, of the Hamiltonian at the time of copying [9]; i.e., for a copy from a source site $\vec{x} \prime$ towards the target site $\vec{x}$ this becomes,

$$
\Delta H_{\text {chemotaxis }}=H_{\text {after }}-H_{\text {before }}-\chi \delta\left(\sigma_{\vec{x}}, 0\right)\left(c_{V}(\vec{x})-c_{V}\left(\vec{x}^{\prime}\right)\right),
$$

with $H_{\text {after }}$, the value of the Hamiltonian after the potential copying step and $H_{\text {before }}$, the original value.

Two partial-differential equations describe the density of ECM material, $c_{E}$, and the concentration of ECM-degrading (proteolytic) enzymes, $c_{M}$. The ECM-degrading enzymes digest the ECM according to first-order kinetics at the sites not covered by any cells. The endothelial cells produce a diffusive ECM-degrading enzyme, $c_{M}$, at a rate proportional to the VEGF concentration. The key new insight of this model includes the way how the ECM can orchestrate the formation of a continuous sprout by coordinating the behavior of the endothelial cells, as illustrated in Figures 1C-F.

The concentration of ECM can modulate the motility of cells, a phenomenon known as haptokinesis [10], with cells obtaining the highest velocity at an optimal ECM concentration. At lower concentrations of ECM, the cells are thought to get insufficient grip on the ECM to pull themselves forwards, whereas at higher concentrations the cells would be bound too tightly to the ECM to move optimally.

In analogy with the chemotaxis term (Eq. 3) we modeled haptokinesis by reducing the probability of sprout extension into the CPM at suboptimal and superoptimal concentrations,

$$
\Delta H_{\text {haptokinesis }}=-\eta \delta\left(\sigma_{\vec{x}, 0}\right)\left(-1+\frac{1}{\rho \sqrt{2 \pi}} e^{-\frac{\left(c_{E}\left(\vec{x}^{\prime}\right)-\mu\right)^{2}}{2 \rho^{2}}}\right),
$$

where $\eta$ is the haptokinesis strength, $\mu=0.5$ is the intermediate ECM density (with $c_{E} \in[0,1]$,) and $\rho=0.2$ is a standard deviation ${ }^{1}$.

\footnotetext{
${ }^{1}$ In the original version of the model [5], a coding error in the haptotaxis term reduced extensions at cell-cell interfaces, but the model still behaves as presented after correcting the mistake.[11]
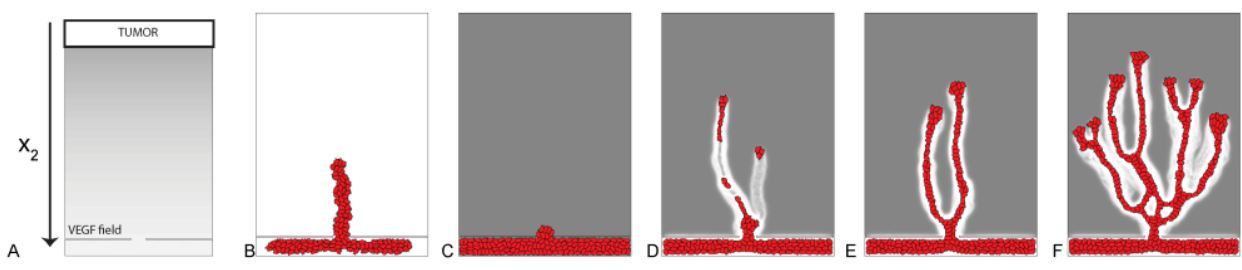

Figure 1. Simulated blood-vessel due to chemical cell-matrix interactions [5]. Morphology at 35000 MCS. (A) simulation set-up; (B) cell migration towards tumor; (C) blocked cell migration in high concentrations of ECM; (D) cell migration with ECM breakdown; (E) sprout with cell proliferation; (F) enhanced branching due to haptotaxis. Modified after Fig. 3 of Ref. [5]
} 
We initiated the simulation with a superoptimal ECM concentration of $c_{E}=0.9$, such that the cells do not move without proteolysis (Figure 1C). With proteolysis the cells migrate towards the source of VEGF, and in doing so, they leave behind a trail of the optimal ECM concentration $\left(c_{E}=0.5\right)$ flanked by two suboptimal regions (Figure 1D). As a result, the next cells are more likely to follow the path of the 'leader' (left branch) than to create a new path independently (right branch in Figure 1D). The cells now follow the same path, but they do not yet form a contiguous sprout. In Figure 1E a contact-inhibited proliferation term is added. Apart from chemotaxis, endothelial cells are guided in vitro by gradients of ECM proteins, a process called haptotaxis [12]. In vivo, ECM degradation likely produces such gradients close to the sprout. The implementation of haptotaxis in the CPM is identical to that of chemotaxis, i.e., by substituting $c_{V}$ with $c_{E}$ in Eq. 3. Figure $1 \mathrm{~F}$ shows the effect of the haptotaxis term: It accelerates the invasion of the cells [13] into the ECM, broadening the vascular tree and enhancing the number of sprouts.

\subsection{Mechanical cell-ECM interactions}

Apart from chemical cues, endothelial cells are thought to respond to mechanical cues, a process called mechanotaxis [14]. For example, the response of two adjacent endothelial cells on each other in vitro depends on the stiffness of the substrate that the cells live on [15]. To model such mechanical interactions between endothelial cells and the surrounding extracellular matrix, we have coupled the cellular Potts model to a finite-element model representing the substrate on which cells live [6]. The shape of the cells, as given by the cellular Potts model, determines the forces exerted on the substrate. The finite-element model returns the resulting strains in the matrix, which again influences the shape of the cells.

The Cellular Potts model uses the Hamiltonian as given in Eq. 1 with one small modification: Here the volume constraint takes the deviations of the volume relative to the target volume, as $\lambda((a(\sigma)-A(\sigma)) / A(\sigma))^{2}$. This choice was made in analogy with the engineering strain, but in practice it does not matter. In analogy with the haptotaxis and chemotaxis terms described above, the state of the finite-element model affects the dynamics of the CPM by modifying the value of $\Delta H$ at the time of spin copying,

$$
\Delta H_{\text {durotaxis }}=-g\left(\vec{x}, \vec{x}^{\prime}\right) \lambda_{\text {durotaxis }}\left(h\left(\epsilon_{1}\right)\left(\vec{v}_{1} \cdot \vec{v}_{m}\right)^{2}+h\left(\epsilon_{2}\right)\left(\vec{v}_{2} \cdot \vec{v}_{m}\right)^{2}\right) .
$$

Here $g\left(\vec{x}, \vec{x}_{\prime}\right)=1$ for extensions and $g\left(\vec{x}, \vec{x}_{\prime}^{\prime}\right)=-1$ for retractions, $\lambda_{\text {durotaxis }}$ is a Lagrange multiplier, $\vec{v}_{m}=\widehat{\vec{x}-\vec{x}}$, a unit vector giving the copy direction and $h$ is a sigmoid function; $\epsilon_{1}$ and $\epsilon_{2}$, and $v_{1}$ and $v_{2}$ are eigenvalues and eigenvectors of $\underline{\epsilon}$, i.e., the principal strains and strain orientation.

To calculate the strains around the cells in the CPM, we model the substrate using a twodimensional finite-element model with linear elastic assumptions. The finite element model uses a lattice of the same spacing as the CPM-lattice $\Lambda$, but is shifted over $\left(\frac{1}{2}, \frac{1}{2}\right)$ such that the FEM lattice is evaluated at the lattice nodes, whereas the CPM-lattice is evaluated in the lattice cells. The CPM cells apply a contractile force on the nodes according to a phenomenological model proposed by Lemmon and Romer [16]: the force on each node covered by the cells obeys,

$$
\vec{F}_{i}=\mu \sum_{j}\left(\vec{x}_{j}-\vec{x}_{i}\right)
$$

such that the magnitude of the contractile force between any two nodes $\vec{x}_{j}+\frac{1}{2}$ and $\vec{x}_{j}+\frac{1}{2}$ touching the cell is proportional to their distance. This model generates a net force $F_{i}=0$ at the cells' centroids, and larger contractile forces at cell extensions. The forces $\vec{F}_{i}$ for a given CPM configuration are set as 

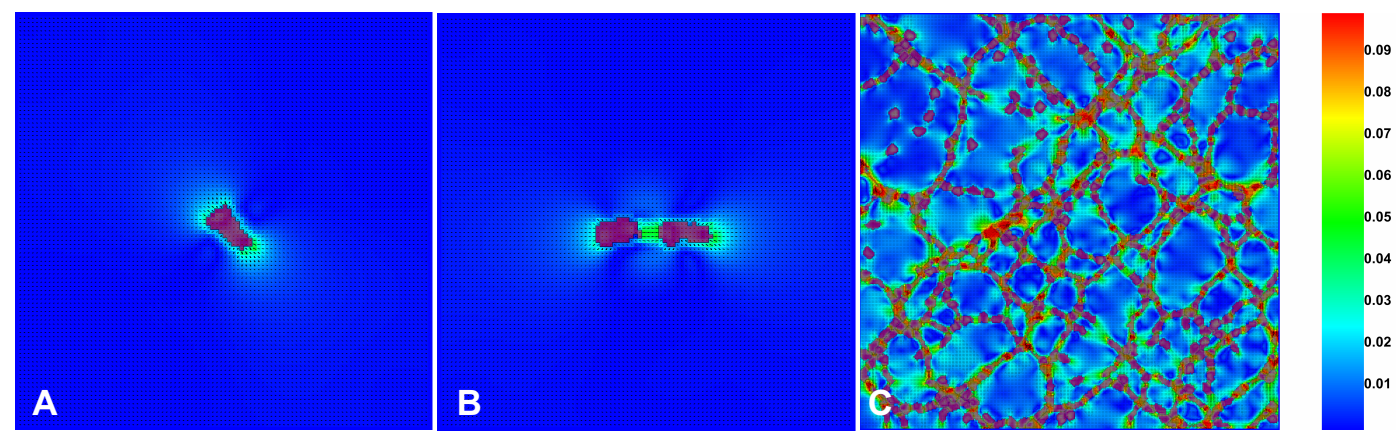

Figure 2. Simulations of mechanical cell-matrix interactions (after Ref. [6]) (A) simulation of a single cell on a $12 \mathrm{kPa}$ substrate at $200 \mathrm{MCS}$ on a $100 \times 100$ lattice; (B) cell pair on a $12 \mathrm{kPa}$ substrate at $500 \mathrm{MCS}$ on a $100 \times 100$ lattice; (C) collection of cells on $12 \mathrm{kPA}$ at $1000 \mathrm{MCS}$ on a $300 \times 300$ lattice. Color bar: magnitude of largest principal strain (unitless)

boundary conditions to the FEM, which is evaluated until (close to) equilibrium. The resulting strain field is then used to evaluate the next cellular Potts iteration.

Figure 2 shows example simulations of the hybrid cell-ECM model on a $12 \mathrm{kPa}$ substrate. In Figure $2 \mathrm{~A}$, single cells spontaneously form a spindle-like shape due to the mechanical feedback with the ECM. As the cell shape fluctuates randomly, the strongest contactile forces occur at cellular protrusions. The resulting strains in the matrix induce the cells to protrude further, which in turns enhances the magnitude of the contractile forces. On matrices much softer than $12 \mathrm{kPa}$, the ECM strains induce protrusion all around the cells, such that the asymmetry is lost. On matrices that are stiffer than $12 \mathrm{kPa}$, the resulting ECM strains do not suffice to induce additional protrusions. These simulation results resemble experimental observations of cardiac myocytes on compliant matrices [17]: On soft matrices the cells contract, on glass substrates the cells spread out, and on matrices of intermediate stiffness the cardiomyocytes form spindle shapes. As a clear discrepancy with these results, however, in our model the (modified) area constraint keeps the cells from spreading out on stiff substrates. This discrepancy may be accounted for by combining our model with a recently proposed extension of the CPM [18] that maximizes the number of cell-substrate adhesions.

Interestingly, our model results suggest that the strains that the cells generate locally in the ECM may also suffice to coordinate the shape and motility of adjacent cells. Figure 2B shows an example of such shape coordination. The strains generated by cells induces cellular protrusions in adjacent cells, thus keeping the cells together. The model also predicts that the assumed cell-ECM feedback at the level of individual pseudpod suffices to co-align cells in a head-to-tail fashion. Figure $2 \mathrm{C}$, finally, shows how these assumption suffice to coordinate cell orientation collectively. The cells tend to organize into network-like structures.

\section{Discussion and Outlook}

In this short article, we have briefly reviewed two of our previous, cell-based mathematical models of ECM-mediated coordination of collective cell behavior during angiogenesis. The first model gave an example of chemical modification of the ECM by means of proteolysis, whereas the second model provided an example of mechanical cell-cell communication. Although the mechanical model has 
been validated against cell culture data [6], both approaches of course only scratch the very surface of the complexity of angiogenesis; they should therefore be seen as proof of principle studies.

What are the next steps? Some of our previous models $[1,2]$ have considered the possibility that endothelial cells attract one another by secreting a chemoattractant, e.g., vascular-endothelial growth factor (VEGF). This proposal is under debate, because VEGF diffuses more quickly than required for such a mechanism. Recent work has proposed a role for the extracellular matrix in this. Matrix components accumulating nearby endothelial cells, act to spatially restrict VEGF around the endothelial cell, resulting in sufficiently steep local chemoattractant gradients that suffice for the formation of vascular networks [19]. In this light, we are currently extending a variant of the "chemical" ECM model [20] based on an angiogenesis cell culture system to include the interaction of matrix-degrading enzymes with secreted growth factors; in such a model the endothelial cells have a fine-level control of the growth factors they "see" and bind to in the microenvironment.

The models presented here studied the collective behavior of one type of endothelial cell, whereas angiogenic sprouts in vivo are led by specialized migratory endothelial cells, called the tip cells, whose fate is specified by a Dll4-Notch-VEGF signaling network. Our more recent models include this signaling network in each of the endothelial cells, in order to study by what mechanisms tip cells can contribute to angiogenesis, e.g, by accelerating sprout progression [21]. In vivo, the tip cell is regularly replaced by trailing endothelial cells in the sprout, a phenomenon called "tip cell overtaking" [22]. In our models such tip cell overtaking can occur as a side effect of sprout extensions [23], suggesting the possibility that tip cell overtaking is non-functional and that Dll4-Notch signaling acts to ensure that the cell at the tip assumes the tip cell phenotype, as opposed to a model where the tip cell actively migrates to the tip.

The above extensions study the mechanical and chemical cell-cell interaction hypotheses in isolation from one another, but it is likely that during angiogenesis they act in concert, and that mechanics affects chemical signaling and vice versa. We are currently exploring the implications of such mechanochemical signaling in our models. This will give us a more complete view of the ways that the ECM can mediate intercellular signaling, and would potentially lead the way towards hypotheses for new treatment options. From a mathematical point of view, these cell-based, mechanochemical models will open up a fascinating class of cell-based models that have only just started to be explored.

\section{Acknowledgments}

Lisanne Rens is thanked for running the simulations in Figure 2. The investigations were supported by the Division for Earth and Life Sciences (ALW) with financial aid from the Netherlands Organization for Scientific Research (NWO).

\section{References}

[1] R.M.H. Merks, S.V. Brodsky, M.S. Goligorksy, S.A. Newman, J.A. Glazier, Dev. Biol. 289, 44 (2006)

[2] R.M.H. Merks, E.D. Perryn, A. Shirinifard, J.A. Glazier, PLoS Comp. Biol. 4, e1000163 (2008)

[3] M.M. Palm, R.M.H. Merks, Phys. Rev. E, 87, 012725 (2013)

[4] D. Palachanis, A. Szabó, R.M.H. Merks, Comp. Part. Mech., Online first (2015)

[5] J.T. Daub, R.M.H. Merks, B. Math. Biol. 75, 1377 (2013)

[6] R.F.M. van Oers, E.G. Rens, D.J. LaValley, C.A. Reinhart-King, R.M.H. Merks, PLoS Comput. Biol. 10, e1003774 (2014)

[7] A.R.A. Anderson, M.A.J. Chaplain, B. Math. Biol. 60, 857 (1998) 
[8] H. Levine, S. Pamuk, B. Sleeman, M. Nilsen-Hamilton, B. Math. Biol. 63, 801 (2001)

[9] N.J. Savill, P. Hogeweg, J. Theor. Biol. 184, 229 (1997)

[10] S. Palecek, J. Loftus, M. Ginsberg, D. Lauffenburger, A. Horwitz, Nature 385, 537 (1997)

[11] J.T. Daub, R.M.H. Merks, Methods Mol. Biol. 1214, 67 (2015)

[12] G. Davis, D. Senger, Circ. Res. 97, 1093 (2005)

[13] S. Turner, J.A. Sherratt, J.Theor. Biol. 216, 85 (2002)

[14] L. Lamalice, F. Le Boeuf, J. Huot, Circ. Res. 100, 782 (2007)

[15] C.A. Reinhart-King, M. Dembo, D.A. Hammer, Biophys. J. 95, 6044 (2008)

[16] C.A. Lemmon, L.H. Romer, Biophys. J. 99, L78 (2010)

[17] J.P. Winer, A. Chopra, J.Y. Kresh, P.A. Janmey, in Mechanobiology of Cell-Cell and Cell-Matrix Interactions, edited by J.A. Wagoner, B.A.C. Harley (Springer US, Boston, MA, 2011), pp. $11-22$

[18] P.J. Albert, U.S. Schwarz, Biophys. J. 106, 2340 (2014)

[19] A. Köhn-Luque, W. de Back, Y. Yamaguchi, K. Yoshimura, M.A. Herrero, T. Miura, Phys. Biol. 10, 066007 (2013)

[20] S.E.M. Boas, M.M. Palm, P. Koolwijk, R.M.H. Merks, in Mechanical and Chemical Signaling in Angiogenesis, edited by C. Reinhart-King (Springer Berlin Heidelberg, Berlin, Heidelberg, 2012), pp. 161-183

[21] M.M. Palm, M.G. Dallinga, E. van Dijk, I. Klaassen, R.O. Schlingemann, R.M.H. Merks, arXiv: 1409.5895

[22] L. Jakobsson, C.A. Franco, K. Bentley, R.T. Collins, B. Ponsioen, I.M. Aspalter, I. Rosewell, M. Busse, G. Thurston, A. Medvinsky et al., Nat. Cell Biol. 12, 943 (2010)

[23] S.E.M. Boas, R.M.H. Merks, BMC Syst. Biol., in press. arXiv:1507.06230 